\title{
Políticas epistémicas y gobernanza. Un análisis comparativo de las regulaciones norteamericana y europea sobre declaraciones de propiedades saludables
}

\author{
Epistemic policies and governance. A comparative analysis \\ of the North American and European health claims regulations
}

\section{NOEMÍ SANZ-MERINO}

Universitat de les Illes Balears

JOSÉ LUIS LUJÁN

Universitat de les Illes Balears

\section{Cómo citar/Citation}

Sanz-Merino, N. y Luján, J. L. (2021). Políticas epistémicas y gobernanza. Un análisis comparativo de las regulaciones norteamericana y europea sobre declaraciones de propiedades saludables. Revista Española de Ciencia Política, 55, 93-117. Doi: https://doi.org/10.21308/recp.55.04

\section{Resumen}

Este artículo analiza las regulaciones norteamericana y europea sobre las declaraciones de propiedades saludables (health claims). Haciendo uso del concepto de política epistémica, mostramos que la regulación europea está comprometida con un enfoque evaluador monista, que privilegia una determinada metodología científica, mientras que la regulación norteamericana está comprometida con una política epistémica pluralista. El análisis comparativo de ambas regulaciones muestra que cada política epistémica depende de los objetivos reguladores. Concluimos que no existe una única política epistémica que sirva para alcanzar distintos objetivos reguladroes y también que la elección de una política epistémica u otra atrinchera los objetivos de la regulación, fija la dirección de la innovación tecnológica y posee otras implicaciones sociopolíticas. En concreto, mostramos cómo la política epistémica europea es compatible con un modo de gobernanza duro (hard governance), mientras que la norteamericana es más compatible con un modo más suave (soft governance). Finalmente, defendemos que nuestras conclusiones son extensibles a las propuestas actuales de políticas basadas en la evidencia. No es razonable, en correspondencia con los resultados obtenidos, pensar que hay un tipo de evidencia científica útil para todos los objetivos de las políticas públicas. Comprometerse con 
una política epistémica u otra conduciría a pensar que solo cierto tipo de intervenciones políticas podrían contar con evidencia que las justifique.

Palabras clave: declaraciones de salud, alimentos, regulación, política basada en evidencia, ciencia reguladora, gobernanza, Europa, Estados Unidos.

\begin{abstract}
This article analyzes North American and European health claims regulations. By making use of the concept of epistemic policy, we show that the European regulation is committed to a monistic assessment approach, which favors a certain scientific methodology, while the North American regulation is committed to a pluralist epistemic policy. The comparative analysis of both regulations shows that each epistemic policy depends on particular regulatory objectives. We conclude that there is no single epistemic policy that serves to achieve different regulatory objectives. Moreover, we conclude that the choice of one epistemic policy or another entrenches the objectives of regulation, sets the trajectory of technological innovation and it entails other socio-political implications. In particular, we show how the European epistemic policy is compatible with a hard governance mode, while the North American one is more compatible with a softer governance mode. Finally, we hold that our conclusions are extensible to current evidence-based policy proposals. It is not reasonable, in accordance with our results, to think that there is one single type of scientific evidence useful for all public policies objectives. Committing to one or another epistemic policy would lead to think that only a certain type of political interventions would have supporting evidence.
\end{abstract}

Keywords: health claims, food, regulation, evidence-based policy, regulatory science, governance, Europe, United States.

\title{
INTRODUCCIÓN
}

Las declaraciones de propiedades saludables afirman un beneficio específico de cierto alimento sobre un aspecto concreto de la salud humana o la prevención de alguna enfermedad. Por ejemplo, mientras que «sin azúcares ańadidos» es una declaración nutricional habitual, el reclamo «reduce el colesterol» en un etiquetado es una declaración de propiedades saludables (o «declaración de salud»). Antes de la regulación de estas declaraciones, presentes hoy en el etiquetado de algunos alimentos de consumo general, se utilizaba la expresión «alimentos funcionales» porque dicho beneficio se agrega a las funciones habituales de nutrición y sabor de los alimentos. Hoy en día, hablar de alimentos funcionales incluye una gran variedad de productos. Aquí solo nos ocuparemos de aquellos alimentos que la regulación actual admite que sean etiquetados con declaraciones sobre propiedades saludables (health claims).

En Europa, la posibilidad de comercializar productos alimentarios de acuerdo con esta calificación ha sido una cuestión sujeta a derecho desde 2006 (European Parliament and Council, 2006), mientras que en los Estados Unidos (en adelante EE. UU.) 
lo fue mucho antes, desde 1990 (US-FDA, 1990). En ambos casos, se establece que el uso de etiquetas de alimentos que contengan este tipo de declaraciones deberá haber sido aprobado de acuerdo con el dictamen positivo previo de un órgano de evaluación científica. La actividad de tales organismos expertos se conoce como «ciencia reguladora» (Jasanoff, 1990): actividad científica cuyo objetivo es generar conocimiento que pueda usarse en el diseńo y establecimiento de políticas públicas y/o ayudar en la toma de decisiones reguladoras concretas. La ciencia reguladora de este tipo de etiquetado es una instancia de la evaluación de beneficios (benefits assessment) (Bidlack et al., 2009; Aggett et al., 2005), al igual que la actividad científica encargada de analizar la efectividad de los fármacos.

La regulación del uso de declaraciones de salud ha sido objeto de investigación desde varias perspectivas. Algunos trabajos se han centrado en el estudio del desarrollo, implantación y evolución de esta regulación y/o las distintas metodologías de evaluación utilizadas, incluyendo en algunos casos comparaciones internacionales $(v$. g. Asp y Bryngelsson, 2008; Sánchez y Casilli, 2008; Gilsenan, 2011; Lalor y Wall, 2011; Tijhuis et al., 2012; Boobis et al., 2013; Hart et al., 2013; Bagchi, 2014; González Díaz et al., 2018; Domínguez Díaz et al., 2020a, 2020b). También se han realizado estudios antropológicos, de enfoque ético y, cada vez más, relativos a las actitudes y percepción de los consumidores (véanse las revisiones de estudios disponibles de Berhaupt-Glickstein y Hallman, 2017; Hieke y Grunert, 2018).

Nuestro propósito general es analizar la interacción entre la actividad científica y los objetivos de las regulaciones, así como sus posibles consecuencias no científicas. A este respecto, realizaremos un estudio comparativo de las regulaciones estadounidense y europea sobre la autorización del uso de declaraciones de salud.

Comenzaremos definiendo los conceptos clave del marco teórico propuesto para el análisis. Las politicas epistémicas son los enfoques evaluadores que están influidos por consideraciones no únicamente científicas (Luján y Todt, 2018b). Estas políticas epistémicas se relacionan con distintos estilos reguladores más generales que son representativos de modos de gobernanza duro (hard governance) o suave (soft governance) (Radaelli y Meuwese, 2010).

En el tercer apartado se expone la controversia científica existente en torno a cuáles son los estándares de evidencia que deben regir la evaluación de beneficios de alimentos con declaraciones de salud. Ello ayudará a identificar las políticas epistémicas subyacentes a las regulaciones norteamericana y europea, que es el contenido de los apartados cuarto y quinto respectivamente. A continuación, en el apartado sexto, la comparación de ambas regulaciones, con sus correspondientes políticas epistémicas, permitirá identificar sus efectos más allá de los objetivos explicitados en las regulaciones.

Las conclusiones que se deriven del análisis de este caso de ciencia reguladora trascienden la regulación del etiquetado con declaraciones de salud y podrían extrapolarse al ámbito general de las políticas públicas basadas en la evidencia. En los últimos tiempos se ha producido un debate en torno a las propuestas de este tipo de políticas públicas en similares términos (Cartwright y Hardie, 2013; Cartwright y Stegenga, 2011). Estas propuestas están relacionadas con diferentes ámbitos de interés público: 
educación, salud, desarrollo, crimen y violencia, mercado laboral, etc. Como finalmente argumentaremos, en tanto que elegir una política epistémica conlleva distintas consecuencias sociales, políticas, científicas, sobre innovación, etc., estas han de ser consideradas al optar, mediante la regulación, por una política epistémica particular.

\section{CIENCIA REGULADORA, POLÍTICAS EPISTÉMICAS Y GOBERNANZA}

Desde finales de los años sesenta del siglo $\mathrm{xx}$, las aplicaciones tecnológicas y/o sus efectos han sido objeto de regulación por parte de Gobiernos y Parlamentos ${ }^{1}$. Ejemplos de este tipo de regulaciones son las relativas a los riesgos de los productos tóxicos, a los efectos de los productos farmacéuticos, las regulaciones medioambientales, etc. En general, son normativas cuyo objetivo suele ser la protección de la salud pública y/o del ambiente (Luján y López Cerezo, 2004).

La regulación de las aplicaciones científico-tecnológicas requiere de conocimiento científico respecto de posibles impactos, dosis, pervivencia de los residuos, etc. Debido a ello se desarrolla paralelamente una actividad científica que Jasanoff (1990) ha llamado «ciencia reguladora». Se trata de una actividad científica aplicada, cuyo objetivo es obtener conocimiento útil para elaborar regulaciones o tomar decisiones políticas concretas. Las evaluaciones de riesgos, de tecnologías o de impacto ambiental serían ejemplos de esta ciencia reguladora.

La ciencia reguladora se diferencia de la ciencia académica tanto por el contexto como por el contenido (ibid.). El contexto de la regulación está politizado, hay intereses económicos, participan grupos de opinión y defensores de alguna causa, etc. Respecto de la práctica científica, las normas epistémicas son más difusas en la ciencia reguladora que en la ciencia académica. Otras diferencias tienen que ver con el contenido, en tanto que la ciencia reguladora se ha de ocupar de rellenar ciertas lagunas de conocimiento para poder asesorar con diligencia en la elaboración de regulaciones y políticas públicas.

Desde una perspectiva normativa, las posiciones respecto a la relación entre ciencia académica y ciencia reguladora las podemos agrupar en dos bloques. Para algunos analistas, las normas epistémicas de la ciencia reguladora han de ser las mismas que las de la ciencia académica, que ha de servir como modelo (véanse, por ejemplo, Laudan, 1984, 2004; Dorato, 2004; Mitchell, 2004; Betz, 2013; Cox, 2015). Para otros, la ciencia reguladora puede seguir normas epistémicas distintas para cumplir mejor con el objetivo de proporcionar conocimiento útil para la regulación y la toma de decisiones (véanse, por ejemplo, Cranor, 1995, 1997; Wandall, 2004; Douglas, 2009; Steel, 2010).

La presente investigación consiste en un estudio de caso de ciencia reguladora: la interacción entre la actividad científica y la regulación del etiquetado de los alimentos

1. Se crearon también en ese momento las agencias reguladoras, especialmente en los EE. UU. 
con propiedades saludables (health claims). Nuestros objetivos específicos son identificar y comparar las políticas epistémicas subyacentes a estas regulaciones en el caso de los EE. UU. y la Unión Europea, las motivaciones no epistémicas que podrían haber conducido a su establecimiento y evolución, así como algunas de las posibles implicaciones o efectos de su implantación.

El contexto analítico más general de este trabajo es, por tanto, el estudio de la interrelación entre valores (o factores) epistémicos y no epistémicos y, en particular, la influencia de estos últimos sobre las decisiones metodológicas en el ámbito de la ciencia reguladora (Jasanoff, 1990, 2005; Shrader-Frechette, 2004, 2010; Stirling, 2007; Cranor, 2006, 2017; Haack, 2014; Luján y Todt, 2015; Whittaker, 2015). Es en este sentido en el que hacemos uso del concepto de política epistémica, en línea similar a lo planteado por Luján y Todt (2018a), Jukola (2019) y Sanz Merino (2021).

Las políticas epistémicas responden a varias cuestiones: determinan el tipo de conocimiento pertinente estableciendo la carga de la prueba; fijan los requisitos de justificación de las afirmaciones de conocimiento mediante el estándar de prueba, y optan por una jerarquía evidencial particular, especificando el valor de distintos tipos de información científica. Las políticas epistémicas se pueden analizar en la regulación y/o en la ciencia reguladora. En este último ámbito se traducen en decisiones metodológicas.

La elección de las políticas epistémicas está determinada por distintos factores, y no exclusivamente los que tienen que ver con cuestiones puramente científicas (factores o valores epistémicos). Influyen también factores relacionados con la importancia relativa que se le otorgue, por ejemplo, a la protección del entorno o de la salud pública, al apoyo a la innovación tecnológica, etc. Factores estos que dependen de los climas sociales imperantes en cada momento ${ }^{2}$.

Pero la puesta en práctica de investigaciones reguladoras particulares (basadas en los enfoques evaluadores derivados de las políticas epistémicas elegidas) también tiene consecuencias no epistémicas: por ejemplo, efectos que pueden ir más allá de los directamente perseguidos por la regulación (en este caso, $v$.g., más allá de velar por la salud de la población). Esto ha sido así respecto de la posible participación de la ciudadanía en la producción, uso e interpretación del conocimiento base de políticas reguladoras; en el caso de la regulación biotecnológica (Jasanoff, 2005), o en el de la evaluación de las declaraciones de salud (Sanz Merino, 2020, 2021).

En este sentido, analizamos la influencia de las políticas epistémicas sobre la implantación, por parte de la Administración estadounidense y de la Unión Europea, de dos modos alternativos de gobernanza: blando o duro (soft regulation/

2. Las regulaciones actuales son en general más protectoras de la salud pública y del entorno que las de hace algunas décadas. Esto ha conducido a que, por ejemplo, la actual regulación europea de los productos químicos pueda interpretarse como una regulación que ha invertido la carga de la prueba desde la Administración a los productores. Esto constituye claramente un cambio de política epistémica. 
hard regulation) $)^{3}$. Partimos de una definición operativa que creemos recoge los rasgos esenciales de las clasificaciones propuestas por otros autores en contextos similares ${ }^{4}$. Una regulación dura es aquella que establece normas o criterios de manera categórica, sin que su aplicación pueda modularse según circunstancias contextuales. Por ejemplo, son instancias típicas el derecho positivo y los marcos legales o normativos vinculantes y generales (hard law), según los cuales, además, los asuntos o procesos objeto de regulación se entienden como acabados o definidos según estándares incuestionables.

En cambio, una regulación suave o blanda permite la intervención u opinión de diversos actores bajo ciertas circunstancias, según criterios menos rígidos, tales como el cumplimiento de procedimientos concretos o la provisión de determinada información. Este enfoque regulador considera los procesos de acción o toma de decisiones como inacabados o susceptibles de ser abiertos en circunstancias concretas, y suele responder al establecimiento de directrices, recomendaciones o marcos normativos flexibles (soft law).

\section{DECLARACIONES DE PROPIEDADES SALUDABLES Y EVIDENCIA CIENTÍFICA}

Tanto en los EE. UU. como en Europa, la regulación de las declaraciones de salud hace referencia al tipo de conocimiento que fundamenta las respectivas decisiones administrativas. La regulación norteamericana se refiere al «acuerdo científico significativo" (US-FDA, 1990) y la europea a la "evidencia científica generalmente aceptada» como más alto estándar evidencial (European Parliament and Council, 2006). Ambas regulaciones, por tanto, parten de la base de la existencia de evidencia científica "generalmente aceptada» o que genera «acuerdo científico». La realidad, no obstante, es que ambas regulaciones han producido importantes controversias respecto de la evidencia pertinente y las metodologías adecuadas.

Actualmente, la evidencia que en ambos casos se identifica explícitamente con el más alto estándar de substanciación científica es la relativa a la relación causal entre un

3. Este interés puede encontrarse en el trabajo de Radaelli y Meuwese (2010), aunque sobre la evaluación de impactos (i. e. la evaluación de eficacia y efectos de actividades organizativas o de políticas públicas) de las medidas de coordinación administrativa y para el aprendizaje organizacional de la Unión Europea. En él se muestra que no solo las políticas diseñan e implementan instrumentos de gestión o regulación, sino que en su puesta en marcha los instrumentos generan sus propios efectos políticos. Para un análisis más general sobre la deriva de las políticas públicas recientes de la Unión Europea según criterios de gobernanza dura y blanda, véase Dehousse (2016).

4. Además de las referencias ya señaladas en la nota anterior, pueden tomarse también ejemplos de definición y uso de la dicotomía de Rapp et al. (2011); Maggetti (2015); Knudsen (2018), o Oberthür (2019). 
alimento o sustancia nutricional (natural o artificial) y un beneficio específico para la salud (EFSA-NDA, 2017; US-FDA, 2009). Este tipo de evidencia es muy difícil de obtener si no es a través de una metodología científica concreta, a saber, las pruebas controladas aleatorizadas (radomized control trials o RCT). Esta es precisamente la metodología que ambas normativas consideran que ofrece la mejor evidencia posible.

Desde su implantación, ambas regulaciones han generado desacuerdos dentro y fuera de la comunidad científica. En los EE. UU. los problemas más visibles surgieron por el enfrentamiento de los solicitantes (productores alimentarios) con la Administración. Según los solicitantes de las declaraciones, había una falta de definición clara en la normativa acerca de qué evidencia científica formaba parte del referido «acuerdo significativo» (véase US DC Circuit, 1999). En aquel momento, la normativa no hacía referencia explícita a los estudios RCT.

En el caso de la Unión Europea, la controversia, que se mantiene aún, se centra en la conveniencia de que para la autorización de una declaración se exijan datos procedentes de RCT. Esta controversia permanece en el seno de la comunidad de investigadores en nutrición. Los críticos con la actual regulación europea consideran que es el resultado de una transferencia acrítica de la metodología típica de evaluación de beneficios de los fármacos y que no se han considerado las particularidades de la investigación sobre la alimentación y su efecto en la salud (Gregori y Gafare, 2012). Se argumenta, por ejemplo, que, mientras en los ensayos farmacológicos los RCT suelen ser viables y arrojan datos causales significativos, en el ámbito de la nutrición no solo son muy difíciles de aplicar técnicamente (por lo que son poco habituales), sino que son incapaces de capturar correctamente aspectos que pudieran ser cruciales en el análisis de los beneficios de las dietas (Boobis et al., 2013; Blumberg et al., 2010; Heaney, 2008). Los estudios nutricionales requieren de investigaciones a medio y largo plazo en las que, además, los efectos objeto de estudio son poco pronunciados. Asimismo, se señala que la investigación en nutrición ha de tener también en cuenta la multifuncionalidad natural de los nutrientes, así como su interacción con otros alimentos. Estas y otras características recomiendan, según algunos autores, que no se minusvalore la evidencia proveniente de la investigación epidemiológica o, incluso, de los análisis mecanísticos (Biesalski et al., 2011; Todt y Luján, 2017).

\section{POLÍTICAS EPISTÉMICAS Y DECLARACIONES DE SALUD: EL CASO NORTEAMERICANO}

En los EE. UU., la Nutrition Labelling and Education Act de 1990, que entonces buscaba sobre todo la estandarización general del etiquetado nutricional, otorgó autoridad a la Food and Drugs Administration (FDA) para evaluar y autorizar aquellas etiquetas en las que se afirmara la mejora de la salud mediante alimentos de consumo general. Esta nueva función se hizo efectiva con sus primeras autorizaciones en 1993 (Lalol y Wall, 2011). Desde entonces, la FDA evalúa, con la finalidad de autorizar o no, si las declaraciones de salud solicitadas cumplen con los requisitos evidenciales 
establecidos por la regulación. La polémica ya señalada, relativa a la falta de concreción, junto con la crítica de otros aspectos de esta norma federal, hicieron que se incorporaran cambios fundamentales desde finales de su primera década de vida.

La problemática en torno a la vaguedad del criterio de «acuerdo científico significativo" (scientific significant agreement, SSA), con el que la FDA discriminaba lo que consideraba substanciación científica suficiente para aprobar una declaración, se solventó en parte a través de la litigación civil. Por un lado, con el proceso Pearson vs. Shalala (US DC Circuit, 1999) se determinó que la regulación existente por entonces reducía los derechos de los productores de estos alimentos por incumplimiento de la Cuarta Enmienda a la Constitución, según la cual el Gobierno no puede imponer sanciones o castigos sin aportar razones claras y razonables. Asimismo, en ese proceso judicial, se determinó también que la regulación violaba la Primera Enmienda, es decir, el Gobierno no puede imponerse por ley impidiendo la libre expresión de los ciudadanos estadounidenses. Por otro lado, el hecho de que en la primera década de la regulación pocos productos alimentarios hicieran uso de declaraciones de salud (incluso de las ya aprobadas por la FDA) parecía corroborar que la regulación no resultaba del todo apropiada (Parker, 2003). Con el tiempo, y como primera respuesta a tales acusaciones, la FDA empezó a especificar que la substanciación de las declaraciones debía consistir en la confirmación de causalidad mediante RCT con sujetos humanos. Los objetivos específicos explicitados en la regulación original eran los mismos que precisamente seguiría luego la Unión Europea: proteger al consumidor y fomentar un mercado para la innovación alimentaria (US-FDA, 1990).

La ley federal de 1990 ya había sido modificada antes de 1999; concretamente, la Food and Drug Administration Modernization Act añadió, en 1997, la posibilidad de que otras autoridades científicas, distintas de la FDA, pudieran emitir juicios favorables sobre la validez, para su uso como declaraciones de salud, de los - desde entonces denominados - «health claims based on authoritative statements». Tales «dictámenes autorizados» incluían los realizados por otros organismos gubernamentales o algunas instituciones científicas, como los National Institutes of Health o la National Science Fundation.

Finalmente, en 2003, la FDA Consumer health information for better nutrition initiative estableció una agenda de trabajo que debía ayudar a lograr lo que se convertiría en otro de los objetivos principales de la regulación estadounidense: «Mejorar la disponibilidad pública y la comprensión del consumidor de la evidencia científica más actual sobre cómo las elecciones dietéticas pueden afectar la salud», así como incrementar la competencia en este mercado (US-FDA, 2003). En particular, esta nueva iniciativa debía «aumentar las posibilidades de que los consumidores siguieran dietas más saludables» (ibid.), y para ello:

5. A la apelación de Durk Pearson y Sandy Shaw contra el Departamento de Salud y Servicios Humanos se sumaron la American Preventive Medical Association y Citizens for Health, una organización de consumidores de suplementos dietéticos en pro del cuidado de la salud. 
a) especificar la forma en cómo la FDA debía aplicar el nuevo estándar "peso de las pruebas» (weigh of evidence), establecido con la finalidad de autorizar declaraciones de salud cualificadas (qualifed health claims);

b) establecer un marco regulador con el estatus de ley, que identificase los procedimientos para implementarla y las necesidades de personal y de organización necesarias para aplicarla;

c) desarrollar una agenda para investigar las formas más efectivas de presentar la información científica veraz a los consumidores, así como para identificar los tipos de información que sean engañosos.

Tales propuestas han conducido a una regulación de las declaraciones de salud más flexible que la inicialmente propuesta y que la posteriormente implantada en la UE.

La regulación norteamericana, en su versión actual, concede un lugar central a los consumidores. Hace mayor hincapié que antes en la necesidad de evaluar si la declaración y la información adjunta en el etiquetado son comprendidas por el consumidor medio $^{6}$. Además, al proceso de evaluación de la FDA se le ha incorporado un mecanismo de consulta (panel period) abierto a los comentarios del público general y de los grupos de interés sobre las solicitudes presentadas.

Finalmente, la iniciativa de 2003 también estableció un cambio sustancial en el estándar evidencial (o de prueba), que se mantiene en la actualidad. La señalada apelación de la FDA al desarrollo de un nuevo y detallado marco legal respondía a la necesidad de dar estatuto legal a las recientes subcategorías de declaraciones de salud con ella propuestas. Las «declaraciones cualificadas de propiedades saludables» se autorizan según se ajusten al nuevo estándar evidencial del peso de las pruebas (US-FDA, 2003). La adopción de una política epistémica basada en este, que produce dictámenes graduales (o probabilísticos) y no categóricos, responde a los nuevos objetivos establecidos en la regulación tras la controversia anteriormente seńalada y la Iniciativa de 2003.

Desde entonces, se pueden etiquetar productos alimentarios con declaraciones de salud susceptibles de estar basadas en tres nuevos niveles de evidencia, y que se han ańadido al prevaleciente "acuerdo científico significativo» (SSA), identificado como el de más alto nivel según la jerarquía evidencial ahora establecida. Acorde con las actuales directrices de la FDA (desde 2003; véase, también, US-FDA, 2009), los consumidores son quienes finalmente han de discriminar si el tipo de evidencia científica aportada sobre los posibles beneficios les es suficiente para tomar la decisión de consumir esos alimentos. Para que esto sea posible, los consumidores han de poder comprender no solo la declaración misma, sino también cómo incide en el significado de esta el estándar científico (o nivel de evidencia) que la autoriza (US-FDA, 2003). Es así que, como resultado de la agenda de investigación sobre los consumidores

6. Véase también el Attachment D: Consumer studies research agenda. Improving consumer understanding and product competition on the health consequences of dietary choices (US-FDA, 2003, actualizado en 2018), disponible en: https://cutt.ly/RlzrKA0 (acceso: 23-8-2020). 
mencionada más arriba, se ha dispuesto que las declaraciones cualificadas habrán de ir redactadas de una determinada manera (véase cuadro 1). Puede afirmarse que la regulación norteamericana actual ha tratado de adaptarse a ciertas exigencias tanto de los productores como de los consumidores.

Cuadro 1.

NivelES DE EVIDENCIA ADMITIDOS PARA LAS DECLARACIONES CUALIFICADAS

\begin{tabular}{|c|c|}
\hline $\begin{array}{c}\text { Clasificación } \\
\text { basada en evidencia }\end{array}$ & Lenguaje apropiado para su calificación \\
\hline Segundo nivel & $\begin{array}{l}\text { "Aunque existe evidencia científica que respalda la afirmación, } \\
\text { la evidencia no es concluyente». }\end{array}$ \\
\hline Tercer nivel & $\begin{array}{l}\text { "Alguna evidencia científica sugiere que [...]. Sin embargo, la FDA } \\
\text { ha determinado que esta evidencia es limitada y no concluyente». }\end{array}$ \\
\hline Cuarto nivel & $\begin{array}{c}\text { "Investigación científica muy preliminar y limitada sugiere que }[. . .] \\
\text { La FDA concluye que hay poca evidencia científica que respalde } \\
\text { esta afirmación». }\end{array}$ \\
\hline
\end{tabular}

Fuente: elaboración y traducción propias a partir de la información de US-FDA (2009).

Las «declaraciones de salud» (nomenclatura reservada ahora para las declaraciones según un primer nivel de evidencia, el del acuerdo científico significativo) se siguen juzgando bajo el supuesto de que la mejor evidencia en nutrición es aquella que establece una relación causal (i. e., según resultados de RTC en humanos). Pero la aceptación de declaraciones de salud cualificadas ha abierto la puerta a la consideración, al menos como relativamente relevantes, de datos provenientes de otras metodologías típicas de la investigación nutricional ( $v$. g., estudios epidemiológicos o mecanísticos). Actualmente, «después de evaluar la totalidad de la evidencia científica, la FDA determina si hay un acuerdo científico significativo que respalde la autorización de una declaración de salud, o evidencia creíble para respaldar una declaración de salud cualificada $»^{7}$ (US-FDA, 2009: 7).

Dado que la propia regulación estadounidense aún considera el criterio SSA como el de más alto nivel científico, puede inferirse que ha habido un cambio en los objetivos de la regulación: es preferible, para mejorar la salud pública, una mayor disponibilidad de productos comercializados como saludables, aunque ello suponga la introducción en el mercado de algunos alimentos que posiblemente no lo sean. Se ha producido un cambio de política epistémica: se ha pasado de una cuyo objetivo era minimizar los falsos positivos a una más tolerante con este tipo de error estadístico. El resultado, acorde con los objetivos de la regulación, es un aumento de las posibilidades en la elección de compra del consumidor, a la vez que lo hace responsable de su decisión y de la valoración de la evidencia disponible respecto a las propiedades saludables de los diferentes productos alimentarios.

7. Las cursivas son propias. 
En resumen, la primera versión de la regulación norteamericana desembocó en una controversia respecto a la concreción del estándar de prueba, especialmente entre los productores de alimentos. El resultado de esta controversia fue un cambio de los objetivos de la regulación, que pasan de la protección del consumidor frente a la información engañosa a la promoción de la libertad de elección del consumidor y la libertad de expresión de los productores, si bien ateniéndose a una tipificación de la evidencia disponible. Estos cambios de objetivos de la regulación se traducen en un cambio de política epistémica. Ahora se adopta un enfoque conocido como del peso de las pruebas, que, a su vez, posibilita el uso de metodologías científicas distintas de los RCT.

\section{POLÍTICAS EPISTÉMICAS Y DECLARACIONES DE SALUD: EL CASO EUROPEO}

La Comisión Europea consideró importante la regulación de la comercialización, como tales, de los alimentos con propiedades saludables desde comienzos de este siglo. En 2001 se publicaba el Discussion paper on nutrition claims and functional claims (SANCO/1341/2001), y en 2006 la primera reglamentación europea ya mencionada y que sigue en vigor. Desde entonces, la European Food Safety Authority (EFSA) está a cargo de este tipo de evaluación de beneficios y es la que decide si una solicitud de etiquetado con declaración de propiedades saludables certifica una «evidencia científica suficiente» (European Parliament and Council, 2006). Solo después de la publicación de una evaluación positiva por parte de la EFSA, la autoridad reguladora en este caso (formada por la Comisión - CE-y los Estados miembros) se autoriza el uso de la declaración de salud en el mercado interior de la UE.

El proceso de solicitud de un etiquetado con este tipo de declaración se tramita inicialmente a través de los respectivos Gobiernos nacionales, y luego la ESFA evalúa la solicitud ateniéndose a si la declaración de salud cumple todas y cada una de las siguientes condiciones generales (European Parliament and Council, 2006) ${ }^{8}$ :

1. Que la declaración sea acerca de un beneficio concreto para un grupo poblacional (más o menos amplio), asimismo claramente definido.

2. Que la declaración esté substanciada mediante la evidencia disponible (publicada o no publicada), que siga el más alto estándar científico.

3. Que la declaración sea comprensible para el consumidor medio.

8. Las primera y tercera condiciones coinciden con las mantenidas como condiciones sine qua non para la evaluación de las declaraciones de salud en la regulación norteamericana. Cabe mencionar, sin embargo, que el significado otorgado a "consumidor medio» es distinto en ambas regulaciones (véase, por ejemplo, en Sanz Merino, 2020). 
Una solicitud de declaración de salud, por tanto, no solo ha de incluir un informe con referencias científicas que la avalen, sino que deberá presentarse redactada como se espera que se publique en el etiquetado y acompañada de las especificaciones pertinentes para que el consumo del alimento sea beneficioso y no perjudicial (cuadro 2).

Cuadro 2.

EJEMPLO DE DECLARACIÓN DE SALUD AUTORIZADA POR LA EFSA

\begin{tabular}{ccc}
\hline $\begin{array}{c}\text { Nutriente o sustancia } \\
\text { o categoría alimentaria }\end{array}$ & $\begin{array}{c}\text { Declaración de } \\
\text { propiedades saludables }\end{array}$ & Condiciones de uso \\
\hline & & $\begin{array}{c}\text { La declaración debe ser usada solo para } \\
\text { alimentos que contengan al menos 40mg de }\end{array}$ \\
(DHA) Ácido & $\begin{array}{c}\text { Mantenimiento de la } \\
\text { función cerebral } \\
\text { normal }\end{array}$ & $\begin{array}{c}\text { DHA por cada 100g y 100Kcl. Como apoyo } \\
\text { a la declaración se ha de informar al } \\
\text { consumidor de que el efecto beneficioso se } \\
\text { obtiene con una ingesta de DHA diaria de }\end{array}$ \\
& & $250 \mathrm{mg}$. \\
\hline
\end{tabular}

Fuente: elaboración y traducción propia a partir de la información contenida en EU Register of nutrition and health claims (disponible en: https://cutt.ly/clzi3NI).

La ESFA publica las «opiniones científicas» favorables y desfavorables de su Panel on Dietetic Products, Nutrition and Allergies (NDA) a través del EFSA Journal. Los interesados a los que se les ha denegado una declaración pueden reclamar. El Directorate General Health and Consumers Protection (en nombre de la CE y las autoridades de los Estados miembros) emite respuestas colectivas. Hasta la fecha, las pocas que se han emitido no han puesto en duda las evaluaciones de la EFSA. Por otro lado, cuando una declaración de salud sobre cualquier nutriente (natural o artificial) presente en un alimento ha sido aprobada, esta puede ser usada comercialmente por cualquier empresa de alimentación, siempre y cuando cumpla y siga los términos aceptados por la autorización original ${ }^{9}$, al igual que ocurre en el caso norteamericano.

La regulación europea de las declaraciones sobre propiedades saludables fue planteada con la intención explícita tanto de proteger la inversión en innovación de las empresas alimentarias como, sobre todo, a los consumidores. El hecho de que antes de la regulación los productores de alimentos simplemente las utilizaban como reclamo publicitario, también ponía en peligro la confianza que los consumidores pudieran tener en las declaraciones científicamente justificadas. Así, considerando también la buena imagen social que - los reguladores suponen- poseen los alimentos funcionales y su impacto directo en la calidad nutricional de la dieta de sus consumidores, se estableció que la regulación de las declaraciones de salud debía

9. A pesar de esta diferencia regulatoria con la I+D patentada, la CE basó en parte la necesidad de la regulación sobre declaraciones de salud en la promoción de un marco de competencia comercial justo que, además, fomentara la innovación en alimentación (European Parliament and Council, 2006). 
proteger a los consumidores de información errónea, engañosa o susceptible de malinterpretación relacionada con sus elecciones alimentarias (European Parliament and Council, 2006: 11).

Para asegurar que la ciudadanía pueda confiar en que si decide consumir estos productos mejorará su salud y, en consecuencia, que los productos que llegan al mercado como funcionales lo son realmente, se ha de garantizar la veracidad de las declaraciones. Por lo tanto, los reguladores europeos han asumido que es contraproducente que se aprueben declaraciones sobre productos que realmente no proporcionan los beneficios aducidos. Además, y contrariamente a lo visto en la regulación norteamericana, la norma europea deja claro que evaluar el alcance de la justificación científica de una propuesta de declaración de salud es solo responsabilidad de la EFSA. En el caso europeo, las instancias reguladoras han dejado claro que no interfieren en el trabajo de los evaluadores expertos (de la agencia), o no más allá de imponerles que hagan su trabajo de la mejor manera científica posible (véase Directorate-General for Health and Consumers, 2011). La EFSA ha traducido epistémicamente esta exigencia y los objetivos reguladores adoptando el mayor nivel posible de exigencia evidencial. Como hemos visto en el caso norteamericano, los objetivos de la regulación se traducen en políticas epistémicas particulares.

Algunas características de la política epistémica europea son las siguientes: por un lado, la EFSA exige como condición sine qua non la aportación de pruebas de causalidad (véase EFSA-NDA, 2011, 2017), lo que teóricamente sería un modo de minimizar el número de falsos positivos; es decir, de evitar la aprobación de productos como beneficiosos sin serlo realmente. Por otro, según las exigencias de la EFSA solo los RCT cumplen con el criterio evidencial establecido en la regulación europea. Por lo tanto, podemos caracterizar la política epistémica que respalda este tipo de regulación europea como una postura o enfoque monista y restrictivo ${ }^{10}$. En especial, es altamente exigente a la vez que parcial, sobre todo porque, como se ha expuesto, no hay acuerdo científico respecto de que los RCT sean la metodología que siempre aporta los datos más relevantes en materia de nutrición, es decir, la más útil para la regulación de los alimentos. La existencia de controversia científica respecto a los niveles de evidencia y las metodologías pertinentes justifica la conclusión de que han sido los factores no epistémicos, relacionados con los objetivos reguladores, los que han inducido la elección de la política epistémica.

\section{LAS CONSECUENCIAS DE LAS POLÍTICAS EPISTÉMICAS}

Las diferencias entre las dos políticas epistémicas que acabamos de analizar obedecen principalmente a la existencia de objetivos reguladores distintos. Como

10. Utilizamos aquí la dicotomía entre monismo y pluralismo, a diferencia de Osimani (2020), donde se utiliza para el mismo fin la dicotomía entre elitismo y pluralismo. 
hemos señalado anteriormente, se trata de un ejemplo claro de interacción entre valores epistémicos (los directamente relacionados con la investigación científica) $\mathrm{y}$ valores no epistémicos (los relacionados con los objetivos reguladores) ${ }^{11}$.

Las dos regulaciones comparten el objetivo general de lograr una población más saludable a través de la innovación en productos para la alimentación general y de la generación de nuevos hábitos de consumo. Tanto en la primera versión de la regulación estadounidense como en la europea, este objetivo se tradujo en la imposición del considerado como el estándar científico más alto. A partir de dichas regulaciones, las agencias evaluadoras demandaron evidencia proveniente de RCT en humanos como prueba de substanciación científica de las declaraciones de salud. En ambas jurisdicciones se consideró que permitir la distribución de alimentos etiquetados con declaraciones de salud sin ser realmente beneficiosos podía acarrear un marco de competencia injusto en el mercado alimentario, la pérdida de confianza de los consumidores e, incluso, afectar negativamente a la salud pública. El objetivo era reducir los falsos positivos y los RCT fueron considerados la mejor metodología a tal fin. Se partía, entonces, de que la ciencia reguladora debía ser lo más precisa posible, a imagen y semejanza de la mejor ciencia académica.

El caso norteamericano es especialmente interesante porque muestra claramente cómo el cambio de objetivos reguladores conduce a un cambio de política epistémica. El actual enfoque evaluador en los EE. UU., centrado en el peso de las pruebas (weigh of evidence), es una ampliación de la clásica metodología de descartar los datos científicos no acordes con el estándar más alto (highest standard). Este cambio muestra que otra política epistémica era posible: una política epistémica más pluralista y flexible que se implanta como consecuencia de un cambio de los objetivos reguladores.

Por tanto, con lo tratado hasta aquí, se han alcanzado el primer y segundo objetivos específicos de este trabajo; es decir, hemos identificado, comparativamente, las políticas epistémicas de las regulaciones europea y estadounidense, y hemos establecido las motivaciones no epistémicas que habrían influido en el establecimiento y la evolución de tales enfoques evaluadores. En el resto de este apartado abordaremos los efectos que la implementación de ambas regulaciones, con sus correspondientes políticas epistémicas, conlleva. Las consecuencias son de diferente tipo. A continuación, nos centramos en las siguientes: sobre la investigación en nutrición, la innovación alimentaria, la salud pública y, finalmente, sobre la imposición de estilos de gobernanza duros o suaves (hardlsoft governance).

Respecto de la investigación, adoptar una política epistémica pluralista o monista condiciona la investigación en las ciencias de la nutrición. La política monista transmite el mensaje de que básicamente solo un tipo de evidencia, y por lo tanto solo la metodología científica que lo genera, es relevante en el ámbito de la nutrición. Se

11. Algunas trabajos sobre este tema son Longino (1990); Shrader-Frechette (2005); Douglas (2009); Wilholt (2009); Haack (2014), y Luján y Todt (2015, 2018b). 
deslegitiman, más o menos, otras metodologías científicas. Desde el ámbito regulador, por tanto, se está influyendo en el desarrollo de la investigación de una serie de ciencias relacionadas con la alimentación. El enfoque pluralista es más tolerante con la diversidad metodológica practicada por las diferentes líneas de trabajo en la investigación sobre nutrición (Aggett, 2012; Marks, 2003).

En relación con la innovación, las políticas epistémicas son literalmente ambientes de selección de nuevos desarrollos tecnológicos. Dependiendo de la jerarquía evidencial que se adopte, habrá innovaciones respecto de las cuales será más fácil obtener evidencia relativa a sus efectos beneficiosos sobre la salud. Esto significa que la política epistémica elegida en la regulación se convertirá en un factor determinante de la dirección y del ritmo del cambio tecnológico, aunque en ocasiones ni siquiera se alcanzan los objetivos reguladores perseguidos a este respecto, o no como se esperaría ${ }^{12}$.

Sobre la promoción de la salud se ha argumentado que, al seguir el tipo de política epistémica monista, la UE está asumiendo costes de oportunidad en términos de salud individual y, sobre todo, poblacional, ya que está impidiendo una más amplia circulación pública de información relevante y también científicamente válida sobre los efectos beneficiosos de algunos alimentos (Blumberg et al., 2010) ${ }^{13}$.

La adopción de políticas epistémicas en ámbitos concretos también genera consecuencias sobre el estilo de gobernanza general de una sociedad. Las diferencias actuales, a este respecto, entre las regulaciones aquí analizadas se sintetizan en el siguiente cuadro.

Cuadro 3.

Modos DE GOBERNANZA SEGÚN REGULACIÓN DE DECLARACIONES DE SALUD

\begin{tabular}{lcc}
\hline & Unión Europea & Estados Unidos \\
\hline & Proteger al consumidor & Proteger al consumidor y promover su libre \\
Objetivos de & de la información errónea. & elección informada. \\
la regulación & $\begin{array}{c}\text { Promover la innovación } \\
\text { alimentaria. }\end{array}$ & $\begin{array}{c}\text { Promover la innovación alimentaria } \\
\end{array}$
\end{tabular}

......

12. El estudio de Khedkar et al. (2017) muestra que en la UE no se ha producido un incremento del interés privado por invertir en $\mathrm{I}+\mathrm{D}$ nutricional desde la implantación de la regulación en 2006. Más bien muestra lo contrario, como consecuencia del temor de los productores de alimentos por no cumplir con las exigencias evaluadoras impuestas por la CE. Martinez (2017) apunta a que el reciente incremento en la apuesta comercial por usar declaraciones de salud en los EE. UU. responde a otros factores, especialmente sociales y relacionados con la salud poblacional, además de a la aparición de la regulación de 2003.

13. Si bien, según los estudios de percepción e intención de compra de alimentos con declaraciones cualificadas de salud, es cuestionable que el pluralismo estadounidense esté influyendo positivamente en la generación de nuevos hábitos de consumo en este sentido (véase, $v . g$. en Berhaupt-Glickstein y Hallman, 2017; Hieke y Grunert, 2018). 


\begin{tabular}{|c|c|c|}
\hline & Unión Europea & Estados Unidos \\
\hline $\begin{array}{l}\text { Traducción } \\
\text { epistémica de } \\
\text { los objetivos } \\
\text { reguladores }\end{array}$ & $\begin{array}{l}\text { Los falsos positivos son malos } \\
\text { para el mercado y la sociedad }\end{array}$ & $\begin{array}{l}\text { Maximizar la disponibilidad de opciones de } \\
\text { consumo supone aceptar un número mayor } \\
\text { de falsos positivos }\end{array}$ \\
\hline $\begin{array}{l}\text { Politica } \\
\text { epistémica }\end{array}$ & $\begin{array}{c}\text { «Más alto estándar» } \\
\text { (monismo metodológico) }\end{array}$ & $\begin{array}{c}\text { «Peso de las pruebas» } \\
\text { (pluralismo metodológico) }\end{array}$ \\
\hline $\begin{array}{l}\text { Opciones } \\
\text { Regulación }\end{array}$ & Autorizar/no autorizar & Autorizar/No autorizar/Aceptar otra evidencia \\
\hline
\end{tabular}

\section{Gobernanza dura}

Gobernanza suave

Fuente: elaboración propia.

Al optar por una política epistémica restringida y monista, la Unión Europea estaría implementando un estilo de gobernanza duro; es decir, con esta regulación la UE establece un marco normativo basado en su respaldo a un sistema evaluador que ha impuesto un estándar único, altamente exigente para el campo científico involucrado, e inapelable tanto científica como extra-científicamente. Son única y exclusivamente los expertos gubernamentales los que evalúan las solicitudes y las instancias reguladoras las que aprueban o desaprueban la comercialización de productos con declaraciones en beneficio del mercado y los consumidores. Ni los productores ni los consumidores pueden participar en ese proceso, salvo mediante el cumplimiento de la norma o el consumo de los alimentos que solo los reguladores consideran suficientemente beneficiosos.

La Comisión Europea no se ha cuestionado su enfoque regulador duro en este ámbito, ya sea por considerar que su restringida postura es la mejor manera de proteger a los consumidores y el mercado - como defienden otros especialistas, $v . g$. Edinger (2016) — ya sea por su tradicional énfasis en reestablecer, en general, la confianza de la ciudadanía en las instancias públicas y los sistemas expertos en los que se basan (véase, por ejemplo, CE, 2001).

En cambio, la Administración estadounidense habría evolucionado hacia un estilo de gobernanza más suave en lo que respecta a la regulación de las declaraciones de salud, en tanto que ahora se habilita una mayor participación tanto en los procesos de autorización como en los de evaluación. Por un lado, las autorización y evaluación pueden ser llevadas a cabo por otras instancias científicas distintas de la FDA y el propio proceso evaluador se ha abierto a las consideraciones de distintos actores sobre las solicitudes de declaración. Por otro lado, se ha ampliado el tipo de información científica relevante en el campo de la nutrición, lo que ha resultado en la aceptación de una diversidad de declaraciones. Esto amplía tanto las opciones de compra de los consumidores como las de decisión de los reguladores. Frente a la dicotomía entre autorizar o no una declaración, los reguladores americanos reconocen posibles excepciones a la norma del más alto estándar, basándose en la aceptación de cierta información proveniente de más partes interesadas. 
La elección de una política epistémica a través de la regulación, aunque sea en un ámbito tan específico como el que es objeto de este trabajo, influye en el estilo general de gobernanza. No consideramos que esta ni ninguna de las consecuencias vistas sean necesariamente negativas o positivas, pues son resultado de objetivos políticos concretos. Orientar tanto la investigación y la innovación como la evaluación experta hacia intereses socialmente relevantes es difícilmente criticable. Lo que finalmente queremos señalar es que cuando se ha de optar por una u otra regulación, por una u otra política epistémica, hay que analizar y tener en cuenta las consecuencias de esa opción, que van más allá de los objetivos propios de la regulación. Por ejemplo, la regulación europea en este ámbito contradice algunas propuestas realizadas a principios del presente siglo. Estas incidían en la necesidad de incorporar mecanismos de participación para distintos actores sociales y de reconocer la incertidumbre que implica el uso del conocimiento científico en la toma de decisiones (véanse a este respecto, CE, 2005, 2006, y 2007).

\section{CONCLUSIONES}

La conclusión más importante del presente trabajo es que difícilmente se va a poder generar un único cuerpo de evidencia científica que sea útil para distintos objetivos reguladores. Nuestros estudios de casos muestran que si el objetivo es reducir la presencia de etiquetados engañosos, se necesitará un tipo de evidencia, y si el objetivo es aumentar la presencia en el mercado de productos saludables etiquetados como tales, la evidencia tendrá que ser otra; es decir, tanto las evidencias requeridas en uno y otro caso como las metodologías científicas que las generan son distintas. La evolución de la regulación norteamericana revela que el cambio de objetivos reguladores se traduce en un cambio de política epistémica. Por lo tanto, la utilidad de la evidencia depende de cuáles sean los objetivos reguladores, no siendo posible encontrar políticas epistémicas multipropósito.

La segunda conclusión sería que, además, elegir una determinada política epistémica hace que solo ciertos objetivos reguladores puedan ser alcanzados y que solo determinados problemas puedan ser abordados. Por ejemplo, la centralidad de los RCT en la regulación europea conlleva dos consecuencias importantes: a) hay pocos productos con declaraciones de salud en el mercado, y b) las empresas solo pueden estar motivadas para desarrollar productos con beneficios que puedan ser identificados de acuerdo con la metodología considerada imprescindible por la regulación. Las políticas epistémicas fijan y atrincheran los objetivos reguladores que les dieron origen y orientan la innovación en una línea determinada. Con el tiempo, el principal cuerpo de evidencia será relativo a unos objetivos reguladores particulares y a una cierta línea de innovación en alimentos.

Las decisiones metodológicas asumidas por las instancias evaluadoras también condicionan y construyen diversos escenarios para la gobernanza de este asunto de salud pública, $v . g$., en tanto que implican una mayor o menor involucración de partes 
interesadas en los procesos de decisión. Concretamente, condicionan la posible amplitud de la participación en los procesos de evaluación, autorización y/o consumo de alimentos con declaraciones de salud. En este sentido hemos visto que, mientras la UE se situaría en un modo duro de gobernanza (hard governance), en los EE. UU. la regulación de las declaraciones de salud respondería a uno más suave (soft governance).

Estas conclusiones son extrapolables de la ciencia reguladora a propuestas actuales relativas a la formulación de políticas públicas basadas en la evidencia. La extrapolación es relativamente inmediata. Como acabamos de mencionar, en primer lugar la idoneidad de una política epistémica depende de los objetivos reguladores. En segundo lugar, comprometerse con una política epistémica produce el efecto de que solo determinados problemas sociales podrán ser investigados científicamente y que solo cierto tipo de intervenciones políticas contarán con evidencia que las justifique.

Nuestra conclusión final es que la elección de una política epistémica posee distintos tipos de consecuencias sociales, políticas, científicas, sobre la innovación, etc. Estas consecuencias han de ser analizadas previamente a la elección de una política epistémica particular mediante el establecimiento de la regulación concreta y/o con posterioridad. Utilizar el argumento a priori de que existe una metodología de investigación que es la mejor en cualquier caso y un tipo de evidencia que es el mejor siempre puede resultar en un ocultamiento de las consecuencias de dicha decisión. Esto se aplica tanto a la ciencia reguladora como a las políticas públicas basadas en evidencia.

\section{AGRADECIMIENTOS}

Este trabajo se ha realizado con el apoyo financiero del Fondo Europeo de Desarrollo Regional de la Comisión Europea (FEDER), Ministerio de Ciencia, Innovación y Universidades de España, Agencia Estatal de Investigación (AEI), proyecto de investigación «Estándares de prueba y elecciones metodológicas en la fundamentación científica de las declaraciones de salud» (FFI2017-83543-P), y la ayuda Cas18/00126.

\section{Referencias}

Aggett, Peter J. 2012. «Dose-response relationships in multifunctional food design: Assembling the evidence», International Journal of Food Sciences and Nutrition, 63 (S1): 37-42. Disponible en: https://doi.org/10.3109/09637486.2011.636344.

Aggett, Peter J., Jean-Michel Antoine, Nils-Georg Asp, France Bellisle, Laura Contor, John H. Cummings, John Howlett, Detlef J. G. Müller, Christoph Persin, Loek T. J. Pijls, Gerhard Rechkemmer, Sandra Tuijtelaars y Hans Verhagen. 2005. "PASSCLAIM. Consensus on criteria», European Journal of Nutrition, 44 (supl.1): 5-30. Disponible en: https://doi.org/10.1007/s00394-005-1104-3. 
Asp, Nils-Georg y Susanne Bryngelsson. 2008. «Health Claims in Europe: New Legislation and PASSCLAIM for Substantiation", The Journal of Nutrition, 138 (6): 1210S-1215S. Disponible en: https://doi.org/10.1093/jn/138.6.1210S.

Bagchi, Debasis (ed.). 2014. Nutraceutical and Functional Food Regulations in the United States and Around the World (Second Edition). Londres: Elsevier.

Berhaupt-Glickstein, Amanda y William K. Hallman. 2017. «Communicating scientific evidence in qualified health claims», Critical Reviews in Food Science and Nutrition, 57(13): 2811-2824. Disponible en: https://doi.org/10.1080/10408398 .2015 .1069730 .

Betz, Gregor. 2013. «In defence of the value free ideal», European Journal for the Philosophy of Science, 3: 207-220. Disponible en: https://doi.org/10.1007/s13194-0120062-x.

Bidlack, Wayne R., Diane Birt, Joseph Borzelleca, Roger Clemens, Nicole Coutrelis, James R. Coughlin, George E. Dunaif, Andrew Ebert, Richard Hall, James T. Heimbach, William Helferich, Bernadene Magnuson, Diane B. McColl, Robert S. McQuate, Ian Munro, Barbara Petersen, Ashley Roberts, Joseph Scimeca, Martin Slayne, Thomas Trautman, Rosetta Newsome, Toni Tarver, Cory Bryant, Fred Shank y Steve Taylor. 2009. "Expert Report: Making decisions about the risks of chemicals in foods with limited scientific information", Comprehensive Reviews in Food Science and Food Safety, 8: 269-303. Disponible en: https://doi. org/10.1111/j.1541-4337.2009.00081.x.

Biesalski, Hans K., Peter J. Aggett, Robert Anton, Paul S. Bernstein, Jeffrey Blumberg, Robert P. Heaney, Jeya Henry, John M. Nolan, David P. Richardson, Ben van Ommen, Renger F. Witkamp, Ger T. Rijkers y Iris Zöllner. 2011. «26th Hohenheim Consensus Conference, September 11, 2010. Scientific substantiation of health claims: Evidence-based nutrition», Nutrition, 27: S1-S20. Disponible en: https://doi.org/10.1016/j.nut.2011.04.002.

Blumberg, Jeffrey, Robert P. Heaney, Michael Huncharek, Theresa Scholl, Meir Stampfer, Reinhold Vieth, Connie M. Weaver y Steven H. Zeisel. 2010. «Evidence-based criteria in the nutritional context", Nutrition Reviews, 68: 478-484. Disponible en: https://doi.org/10.1111/j.1753-4887.2010.00307.x.

Boobis, Alan, Alessandro Chiodini, Jeljer Hoekstra, Pagona Lagiou, Hildegard Przyrembel, Josef Schlatter, Katrin Schütte, Hans Verhagen y Bernhard Watzl. 2013. «Critical appraisal of the assessment of benefits and risks for foods. BRAFO Consensus Working Group", Food and Chemical Toxicology, 55: 659-675. Disponible en: https://doi.org/10.1016/j.fct.2012.10.028.

Cartwright, Nancy y Jeremy Hardie. 2013. Evidence-Based Policy: A practical guide to doing it better. Oxford: Oxford University Press. Disponible en: https://doi. org/10.1093/acprof:osobl/9780199841608.001.0001.

Cartwright, Nancy y Jacob Stegenga. 2011. «A theory of evidence for evidence-based policy», en William Twining, Philip Dawid y Dimitra Vasilaki (eds.), Evidence, inference and enquiry. Oxford: Oxford University Press. Disponible en: https:// doi.org/10.5871/bacad/9780197264843.003.0011. 
Comisión Europea (CE). 2001. «La gobernanza europea. Un libro blanco (COM 428)», Diario Oficial C, 287. Bruselas. Disponible en: https://cutt.ly/qlzh3BE.

Comisión Europea (CE). 2005. Science and Society Action Portfolio. Today's science for Tomorrow's Society. Bruselas. Disponible en: https://cutt.ly/Klzjqwm.

Comisión Europea (CE). 2006. Science in Society Programme, From Science and Society to Science in Society Towards a framework for cooperative research. Bruselas: Directorate General Research and Technology Development. Disponible en: https:// cutt.ly/Blzjtmq.

Comisión Europea (CE). 2007. Taking European Knowledge Society Seriously. Report of the Expert Group on Science and Governance to the Science, Economy and Society Directorate. Disponible en: https://cutt.ly/Xlzji0R.

Cox, Louis A. 2015. Breakthroughs in decision science and risk analysis. Hoboken, NJ: John Wiley and Sons.

Cranor, Carl F. 1995. "The social benefits of expedited risk assessment», Risk Analysis, 15:353-358. Disponible en: https://doi.org/10.1111/j.1539-6924.1995.tb00328.x.

Cranor, Carl F. 1997. "The normative nature of risk assessment: Features and possibilities», Risk: Health, Safety and Environment, 8 (2): 123-136.

Cranor, Carl F. 2006. Toxic Torts. Science, Law and the Possibility of Justice. Cambridge: Cambridge University Press. Disponible en: https://doi.org/ 10.1017/CBO9780511617713.

Cranor, Carl F. 2017. Tragic Failures: How and Why We Are Harmed by Toxic Chemicals. Oxford: Oxford University Press. Disponible en: https://doi.org/10.1093/ acprof:oso/9780190635756.001.0001.

Dehousse, Renaud. 2016. "Has the European Union moved towards soft governance?», Comparative European Politics, 14: 20-35. Disponible en: https://doi. org/10.1057/cep.2015.7.

Directorate-General for Health and Consumers. 2011. «2nd collective answer». Disponible en: https://cutt.ly/Rlzjxi3.

Domínguez Díaz, Laura, Virginia Fernández-Ruiz y Montaña Cámara. 2020a. «The frontier between nutrition and pharma: The international regulatory framework of functional foods, food supplements and nutraceuticals», Critical Reviews in Food Science and Nutrition, 60 (10): 1738-1746. Disponible en: https://doi.org/1 $0.1080 / 10408398.2019 .1592107$.

Domínguez Díaz, Laura, Virginia Fernández-Ruiz y Montaña Cámara. 2020b. «An international regulatory review of food health-related claims in functional food products labeling». Journal of Functional Foods, 68: 103896. Disponible en: https://doi.org/10.1016/j.jff.2020.103896.

Dorato, M. 2004. «Epistemic and nonepistemic values in science», en Peter Machamer y Gereon Wolters (eds.), Science, values and objectivity. Pittsburgh: University of Pittsburgh Press.

Douglas, Heather E. 2009. Science, policy, and the value-free ideal. Pittsburgh: University of Pittsburgh Press. Disponible en: https://doi.org/10.2307/j.ctt6wrc78. 
Edinger, Wieke H. 2016. «Promoting Educated Consumer Choices. Has EU Food Information Legislation Finally Matured?», Journal of Consumer Policy, 39: 9-22. Disponible en: https://doi.org/10.1007/s10603-015-9307-3.

European Food Safety Authority. Panel on Dietetic Products, Nutrition and Allergies (EFSA-NDA). 2011. "General guidance for stakeholders on the evaluation of Article 13.1, 13.5 and 14 health claims», EFSA Journal, 9 (4): 2135. Disponible en: https://doi.org/10.2903/j.efsa.2011.2135.

European Food Safety Authority. Panel on Dietetic Products, Nutrition and Allergies (EFSA-NDA). 2017. «Scientific and technical guidance for the preparation and presentation of an application for authorisation of a Health Claim (2 revision)», EFSA Journal, 15 (1): 4680. Disponible en: https://doi.org/10.2903/j. efsa.2017.4680.

European Parliament and Council. 2006. «European Parliament and Council Regulation No. 1924/of the European Parliament and of the Council on Nutrition and Health Claims Made on Foods», OJ L, 404 (30-12-2006): 12. Disponible en: https://cutt.ly/vlzkiKz.

Gilsenan, Mary. 2011. «Nutrition and health claims in the EU: A regulatory overview», Trends in Food Science and Technology, 22: 536-542. Disponible en: https:// doi.org/10.1016/j.tifs.2011.03.004.

González-Díaz, Cristina, Diana Gil-González y Carlos Álvarez-Dardet. 2018. «Scientific Evidence on Functional Food and Its Commercial Communication: A Review of Legislation in Europe and the USA», Journal of Food Science, 83 (11): 27102717. Disponible en: https://doi.org/10.1111/1750-3841.14359.

Gregori, Dario y Claudia Elena Gafare. 2012. «Multifunctional food: Medical evidence and methodological notes on substantiating health claims?», International Journal of Food Sciences and Nutrition, 63 (S1): 29-36. Disponible en: https://doi.org/10.3109/09637486.2011.653553.

Haack, Susan. 2014. Evidence Matters. Science, Proof, and Truth in the Law. Cambridge: Cambridge University Press. Disponible en: https://doi.org/10.1017/ CBO9781139626866.

Hart, Andy, Jeljer Hoekstra, Helen Owen, Marc Kennedy, Marco J Zeilmaker, Nynke de Jong y Helga Gunnlaugsdottir. 2013. «Qalibra: a general model for food risk-benefit assessment that quantifies variability and uncertainty», Food and Chemical Toxicology, 54: 4-17. Disponible en: https://doi.org/10.1016/j.fct.2012.11.056.

Heaney, Robert. 2008. «Nutrients, endpoints, and the problem of proof», Journal of Nutrition, 138: 1591-1595. Disponible en: https://doi.org/10.1093/jn/138.9.1591.

Hieke, Sophie y Klaus G. Grunert. 2018. «Consumers and health claims», en Michele J. Sadler (ed.), Foods, Nutrients and Food Ingredients with Authorised EU Health Claims (vol. 3). Amsterdam: Elsevier. Disponible en: https://doi.org/10.1016/ B978-0-08-100922-2.00002-4.

Jasanoff, Sheila. 1990. The fifth branch: science advisers as policymakers. Cambridge: Harvard University Press. 
Jasanoff, Sheila. 2005. Designs on Nature: Science and Democracy in Europe and the United States. Princeton, NJ: Princeton University Press. Disponible en: https:// doi.org/10.1515/9781400837311.

Jukola, Saana. 2019. "On the evidentiary standards for nutrition advice», Studies in History and Philosophy of Biological and Biomedical Science, 73: 1-9. Disponible en: https://doi.org/10.1016/j.shpsc.2018.05.007.

Khedkar, Sukhada, Stefanie Bröring y Stefano Ciliberti. 2017. «Exploring the Nutrition and Health claims Regulation (EC) No. 1924/2006: What is the impact on innovation in the EU food sector?», Journal of Food sciences and Nutrition, 68 (1): 10-17. Disponible en: https://doi.org/10.1080/09637486.2016.1212818.

Knudsen, Jette S. 2018. "Government regulation of corporate social responsibility: The implications for corporate governance», en Ciaran Driver y Grahame Thomson (eds.), Corporate Governance in Contention. Oxford: Oxford University Press.

Lalor, Fiona y Patrick G. Wall. 2011. "Health claims regulations. Comparison between USA, Japan and European Union», British Food Journal, 113 (2): 298-313. Disponible en: https://doi.org/10.1108/00070701111105358.

Laudan, Larry. 1984. Science and values. Berkeley: University of California Press.

Laudan, Larry. 2004. "The epistemic, the cognitive and the social», en P. Machamer y G. Wolters (eds.), Science, values and objectivity. Pittsburgh: University of Pittsburgh Press.

Longino, Helen. 1990. Science as Social Knowledge. Princeton: Princeton University Press.

Luján, José L. y José A. López Cerezo. 2004. «De la promoción a la regulación. El conocimiento científico en las políticas públicas de ciencia y tecnología», en José Luis Luján y Javier Echeverría (eds.), Gobernar los riesgos. Ciencia y valores en la sociedad del riesgo. Madrid: Biblioteca Nueva.

Luján, José L. y Oliver Todt. 2015. "The role of values in methodological controversies: the case of risk assessment», Philosophia Scientiae, 19 (1): 45-56. Disponible en: https://doi.org/10.4000/philosophiascientiae.1031.

Luján, José L. y Oliver Todt. 2018a. "The dilemmas of science for policy», EMBO Reports, 19 (2): 194-196. Disponible en: https://doi.org/10.15252/embr.201744795.

Luján, José L. y Oliver Todt. 2018b. «Regulatory Science: between Technology and Society», en Belén Laspra y José Antonio López Cerezo (eds.), Spanish Philosophy of Technology. Cham: Springer.

Maggetti, Martino. 2015. "Hard and Soft Governance», en Kennet Lynggaard, Karl Loefgren y Ian Manners (eds.), Research Methods in European Union Studies. Londres: Palgrave Macmillan.

Marks, Harry. 2003. "Rigorous uncertainty: Why RA fisher is important», International Journal of Epidemiology, 32: 932-937. Disponible en: https://doi. org/10.1093/ije/dyg288.

Martinez, Steve. 2017. "Use of health and nutrition-related claims on new food products in the USA from 1989 to 2010», Agro Food Industry Hi Tech, 28 (2): 43-47. 
Mitchell, Sandra. 2004. "The prescribed and proscribed values in science policy», en Peter Machamer y Gereon Wolters (eds.), Science, values and objectivity. Pittsburgh: University of Pittsburgh Press.

Oberthür, Sebastian. 2019. «Hard or Soft Governance? The EU's Climate and Energy Policy Framework for 2030», Politics and Governance, 7 (1): 17-27. Disponible en: http://dx.doi.org/10.17645/pag.v7i1.1796.

Osimani, Barbara. 2020. «Epistemic gains and epistemic games: Reliability and higher order evidence in medicine and pharmacology", en Adam LaCaze y Barbara Osimani (eds.), Uncertainty in pharmacology. Epistemology, methods, and decisions. Cham: Springer. Disponible en: https://doi.org/10.1007/978-3-030-291792_15.

Parker, Betty. 2003. «Food for health. The Use of Nutrient Content, Health, and Structure/Function Claims in Food Advertisements", Journal of Advertising, 32 (3): 47-55. Disponible en: https://doi.org/10.1080/00913367.2003.10639135.

Radaelli, Claudio M. y Anne C. M. Meuwese. 2010. «Hard Questions, Hard Solutions: Proceduralisation through Impact Assessment in the EU», West European Politics, 33 (1): 136-153. Disponible en: https://doi.org/10.1080/01402380903354189.

Rapp, Mark S., Thomas Schmid y Michael Wolff. 2011. «Hard or Soft Regulation of Corporate Governance?», HHL Research Paper Series in Corporate Governance, 6: 1-38. Disponible en: https://dx.doi.org/10.2139/ssrn.1930847.

Sánchez, Sylvie y Antonio Casilli. 2008. «Status and use of food products with health claim (FPHC) in the USA, Japan and France: an anthropological perspective», Food Quality and Preference, 19:682-691. Disponible en: https://doi.org/10.1016/j. foodqual.2008.06.007.

Sanz Merino, Noemí. 2020. «La comunicación social de la ciencia a través del etiquetado de alimentos saludables en los EEUU y Europa», Comunicación. Revista Internacional de Comunicación Audiovisual, Publicidad y Estudios Culturales, 1 (18): 66-84. Disponible en: https://doi.org/10.12795/Comunicacion.2020.i18.04.

Sanz Merino, Noemí. 2021. «Modelos de evaluación de las declaraciones sobre propiedades saludables en alimentos y su impacto en las comprensión y apropiación públicas de la ciencia», Revista Iberoamericana de Ciencia, Tecnología y Sociedad, 16 (46): 149-166.

Shrader-Frechette, Kristin. 2004. «Using Metascience to Improve Dose-Response Curves in Biology: Better Policy Through Better Science», Philosophy of Science, 71: 1026-1037. Disponible en: https://doi.org/10.1086/426772.

Shrader-Frechette, Kristin. 2005. "Objectivity and professional duties regarding science and technology», en Wenceslao González (ed.), Science, Technology and Society: A Philosophical Perspective. A Coruña: Netbiblo.

Shrader-Frechette, Kristin. 2010. «Conceptual Analysis and Special-interest Science», Synthese, 177: 449-469. Disponible en: https://doi.org/10.1007/s11229-0109792-5.

Steel, Daniel. 2010. «Epistemic values and the argument from inductive risk», Philosophy of Science, 77, 14-34. Disponible en: https://doi.org/10.1086/650206. 
Stirling, Andrew. 2007. «Risk, Precaution and Science», EMBO reports, 8: 309-315. Disponible en: https://dx.doi.org/10.1038\%2Fsj.embor.7400953.

Tijhuis, Mariken, Mikko Pohjola, Helga Gunnlaugsdottir, Nikos Kalogeras, O. Leino, Michiel Luteijn, Sveinn Magnússon, Gaby Odekerken, Margherita Poto, Jouni Tuomisto, Øydis Ueland, B. C.White, F. Holm y Hans Verhagen. 2012. "Looking beyond borders: Integrating best practices in benefit-risk analysis into the field of Food and Nutrition", Food and Chemical Toxicology, 50: 77-93. Disponible en: https://doi.org/10.1016/j.fct.2011.11.044.

Todt, Oliver y José L. Luján. 2017. «Health Claims and Methodological Controversy in Nutrition Science», Risk Analysis, 37 (5): 958-968. Disponible en: https://doi. org/10.1111/risa.12665.

United States Court of Appeals, District of Columbia Circuit (US DC Circuit). 1999. «Durk PEARSON and Sandy Shaw, American Preventive Medical Association and Citizens for Health, Appellants, vs. Donna E. SHALALA, Secretary, United States Department of Health and Human Services, et al.», Appellees, 98-5043, 98-5084 (15 de enero). Disponible en: https://cutt.ly/Ylzvq7K.

United States Food and Drug Administration (US-FDA). 1990. Nutrition Labelling and Education Act. Public Law 101-553, 104 Sat. 2353 codified at 21 USC 343 (1993). Disponible en: https://cutt.ly/hlzcbwz.

United States Food and Drug Administration (US-FDA). 2003. Consumer health information for better nutrition initiative: Task Force Final Report. Disponible en: https://cutt.ly/nlzcUYb.

United States Food and Drug Administration (US-FDA). 2009. Guidance for Industry: Evidence-Based Review System for the Scientific Evaluation of Health Claims. Office of Nutrition and Food Labelling. Disponible en: https://cutt.ly/0lzcASk.

Wandall, Birgitte. 2004. "Values in Science and Risk Assessment», Toxicology Letters, 152: 265-272. Disponible en: https://doi.org/10.1016/j.toxlet.2004.05.010.

Whittaker, Margaret. 2015. "Risk assessment and alternatives assessments», Risk Analysis, 35: 2129-2136. Disponible en: https://doi.org/10.1111/risa.12549.

Wilholt, Torsten. 2009. "Bias and values in scientific research", Studies in History and Philosophy of Science, 40: 92-101. Disponible en: https://doi.org/10.1016/j. shpsa.2008.12.005.

Presentado para evaluación: 9 de enero de 2020.

Aceptado para publicación: 10 de enero de 2021.

NOEMÍ SANZ-MERINO

noemi.sanz@uib.es

ORC ID: 0000-0002-5093-3883

Profesora en la Universitat de les Illes Balears. Es doctora en Filosofía y máster en Estudios Sociales de la Ciencia y Políticas Públicas. Ha sido investigadora Torres-Quevedo, 
y ha realizado estancias de investigación en el Consejo Superior de Investigaciones Científicas, la Universidad de Groninga (Países Bajos) y la Universidad de Delaware (EE. UU.), y docente en la Universidad de Oviedo, la Universidad Internacional de Valencia y de distintos programas de la Organización de Estados Iberoamericanos (OEI). Es autora de diversos capítulos de libro, así como de artículos en revistas como Public Understanding of Science, Revista Iberoamericana de Ciencia, Tecnologia y Sociedad, Argumentos de Razón Técnica o la Revista Iberoamericana de Educación.

\section{JOSÉ LUIS LUJÁN}

jl.lujan@uib.es

ORCID: 0000-0002-8829-0609

Catedrático de Lógica y Filosofía de la Ciencia de la Universitat de les Illes Balears. Es doctor en Filosofía por la Universidad de Valencia, y ha sido investigador en la Universidad Pública de Navarra y en el Instituto de Estudios Sociales Avanzados (CSIC). Autor de Ciencia y política del riesgo (con J. A. López Cerezo, 2000); Ciencia, tecnología y sociedad (con M. I. González y J. A. López Cerezo, 1996); La imagen social de las nuevas tecnologías biológicas en España (con J. Atienza, 1997), y editor de Gobernar los riesgos (con J. Echeverría, 2004). Ha publicado en revistas como Futures, Journal of Risk Research, Risk Analysis, European Journal for Philosophy of Science, Social Studies of Science, Science and Public Policy, Studies in History and Philosophy of Science. 